\title{
Plasma sphingolipids: Potential biomarkers for severe hepatic fibrosis in chronic hepatitis $\mathbf{C}$
}

\author{
JUN-FENG LI ${ }^{1,2^{*}}$, FENG QU ${ }^{3 *}$, SU-JUN ZHENG ${ }^{2 *}$, FENG REN $^{2}$, HUI-LI WU ${ }^{2}$, MEI LIU ${ }^{2}$, \\ JIN-YU REN ${ }^{4}$, YU CHEN ${ }^{2}$, ZHONG-PING DUAN ${ }^{1,2}$ and JIN-LAN ZHANG ${ }^{3}$ \\ ${ }^{1}$ The First Clinical Medical School, Lanzhou University, Lanzhou, Gansu 730000; \\ ${ }^{2}$ Artificial Liver Center, Beijing YouAn Hospital, Capital Medical University, Beijing 100069; \\ ${ }^{3}$ State Key Laboratory of Bioactive Substance and Function of Natural Medicines, Institute of Materia Medica, \\ Chinese Academy of Medical Sciences and Peking Union Medical College, Beijing 100050, P.R. China; \\ ${ }^{4}$ Evergreen Wellness Center, Kansas College of Chinese Medicine, Wichita, Kansas 67207, USA
}

Received April 7, 2014; Accepted January 29, 2015

DOI: $10.3892 / \mathrm{mmr} .2015 .3361$

\begin{abstract}
The plasma profile of sphingolipids in hepatic fibrosis patients with chronic hepatitis $\mathrm{C}(\mathrm{CHC})$ is rarely considered at present. The association between plasma sphingolipids and severe fibrosis in $\mathrm{CHC}$ remains an obscure area of research. The aim of the present study was to assess the plasma profile of sphingolipids and to examine the association between plasma sphingolipids and severe fibrosis in $\mathrm{CHC}$, in order to identify potential novel markers of severe fibrosis in CHC. A cohort of 120 treatment-naïve patients with $\mathrm{CHC}$ were included in the present study. Liver biopsies were performed and routine serological indicators were measured. Plasma sphingolipids were detected using high performance liquid chromatography tandem mass spectrometry. A total of 44 plasma sphingolipids were detected. Plasma hexosylceramide (HexCer; d18:1/12:0), HexCer (d18:1/16:0) and HexCer (d18:1/22:0) were shown to be significantly different in patients with $\mathrm{CHC}$ between those with and without severe fibrosis (Metavir $\mathrm{F} \geq 3$; $\mathrm{P}<0.05)$. HexCer (d18:1/12:0) was observed to be closely associated with severe fibrosis in $\mathrm{CHC}$ [odds ratio $(\mathrm{OR})=1.03$ ] following adjustment for confounding variables in a multivariate analysis. HexCer (d18:1/12:0) had
\end{abstract}

Correspondence to: Dr Zhong-Ping Duan, Artificial Liver Center, Beijing YouAn Hospital, Capital Medical University, 8 Xitoutiao, Youwai Street, Beijing 100069, P.R. China

E-mail: duan2517@163.com

Dr Jin-Lan Zhang, State Key Laboratory of Bioactive Substance and Function of Natural Medicines, Institute of Materia Medica, Chinese Academy of Medical Sciences and Peking Union Medical College, 2 Nanwei Road, Beijing 100050, P.R. China

E-mail: zhjl@imm.ac.cn

${ }^{*}$ Contributed equally

Key words: sphingolipids, hepatitis C virus, hepatic fibrosis, lipid, liver biopsy diagnostic value for severe fibrosis in $\mathrm{CHC}$ [area under the curve $(\mathrm{AUC})=0.69$ ]. In patients with $\mathrm{CHC}$ who had developed significant fibrosis (Metavir F $\geq 2$ ), HexCer (d18:1/12:0) remained closely associated with severe fibrosis $(\mathrm{OR}=1.08)$ in this subgroup. In addition, HexCer (d18:1/12:0) had sufficient diagnostic ability $(\mathrm{AUC}=0.73)$ to distinguish severe fibrosis in patients with $\mathrm{CHC}$ with significant fibrosis. In conclusion, the present study indicated that plasma HexCer (d18:1/12:0) exhibits a close correlation with severe hepatic fibrosis in $\mathrm{CHC}$, particularly in patients who have significant fibrosis. Additionally, HexCer (d18:1/12:0) may be a potential marker of severe hepatic fibrosis in $\mathrm{CHC}$.

\section{Introduction}

Hepatitis $\mathrm{C}$ is a disease of global epidemic status, the prevalence of which is $>2 \%$, with $>120$ million individuals infected with the hepatitis $\mathrm{C}$ virus (HCV) (1). Chronic HCV infection may eventually progress to severe fibrosis or cirrhosis, which necessitates surveillance for hepatocellular carcinoma and screening for varices (2). A natural history study revealed that a quarter of patients with chronic hepatitis $\mathrm{C}$ (CHC) with severe liver fibrosis succumbed after a median interval of 3.5 years, although this poor prognosis was improved following combination antiviral treatment (3). Therefore, the evaluation of severe hepatic fibrosis involves assessing the prognosis of, and developing a treatment strategy for, patients with CHC. Although a liver biopsy is the gold standard for assessing the stage of hepatic fibrosis, its clinical application is usually limited due to the invasiveness of this procedure. In addition, factors such as the sampling and observational methods also impact the veracity and the reliability of the results (4-5). Serological markers with easy accessibility, are the primary factor used to assist in evaluating liver fibrosis. However, the insufficient validation of their function means they are inadequate for accurately monitoring changes in the stages of fibrosis in CHC (4). Therefore, it is necessary to identify novel and effective noninvasive markers for the diagnosis and treatment of hepatic fibrosis, particularly for severe fibrosis in $\mathrm{CHC}$. 
Sphingolipids, consisting of a sphingosine backbone, are fundamental structural components of cell membranes and incorporate other constituents in order to form lipid rafts (6). Certain receptors or kinases are associated with lipid rafts, which form platforms that function in signaling and trafficking on the plasma membrane, and sphingolipids are involved in the regulation of signaling pathways in cell growth, differentiation and apoptosis $(7,8)$. During HCV infection, the HCV RNA level may be significantly reduced if the gathering of non-structural proteins of $\mathrm{HCV}$ on lipid rafts is disrupted via the inhibition of serine-palmitoyltransferase, a key enzyme in the de novo synthesis of sphingolipids $(9,10)$. Thus, host sphingolipids may impact upon the infection process of $\mathrm{HCV}$ and may reflect the disease status of the HCV infection. In addition, sphingolipids also have the potential to affect the pathogenesis of tissue fibrosis (11). Sphingosine 1-phosphate (S1P), as a bioactive lipid mediator, is involved in numerous signaling pathways and regulates a wide variety of cellular functions (12). It has been shown that the signaling axis with which S1P is involved, exerts a powerful migratory effect on hepatic myofibroblasts and is involved in the development of hepatic fibrosis (13). Therefore, it is hypothesized that sphingolipids may be associated with hepatic fibrogenesis. Although a clinical study looking at a single sphingolipid in $\mathrm{CHC}$, in the case of S1P declining in CHC patients has been previously reported (14), the study of the full plasma sphingolipid profile in hepatic fibrosis induced by HCV has not been previously investigated, to the best of our knowledge, and little is currently known regarding the diagnostic value of plasma sphingolipids.

A mature high-performance liquid chromatography-tandem mass spectrometry (HPLC-MS/MS) method was previously established (15), and has been employed to identify an association between plasma sphingolipids and hepatic inflammation in $\mathrm{CHC}$, using improved quantitative high-throughput lipidomic platform (16). The present study, based on liver biopsies, analyzed alterations in the plasma profile of sphingolipids of a cohort of untreated patients with CHC, with and without severe fibrosis, using HPLC-MS/MS. This approach was intended to identify the plasma sphingolipids that are associated with the development of severe fibrosis, in particular, severe fibrosis in patients with $\mathrm{CHC}$ who have developed significant fibrosis (Metavir $\mathrm{F} \geq 2$ ).

\section{Patients and methods}

Patients. A cohort of 122 patients from Dingxi (Gansu, China) were enrolled in the present study at Beijing YouAn Hospital, Capital Medical University (Beijing, China) between July 2010 and June 2011. All patients had a history of paid plasma donation between 1992 and 1995. The diagnosis of CHC was made in accordance with previously described criteria $(2,17)$. Other viral co-infections, including the hepatitis B virus, or other liver diseases were excluded. No patients had received antiviral therapy prior to enrolment in the present study. Two patients were excluded from the study due to the collection of an invalid specimen from the liver biopsy or due to the presence of ascites unsuitable for puncture, as this increase the risk of intra-abdominal infection during biopsy. Thus, 120 patients were eligible for the study. Based on the liver biopsy, 64 patients with significant hepatic fibrosis $(F \geq 2)$ were eligible for subgroup analysis.

Blood from the cubital veins of fasting patients was collected on the day of the biopsy. Routine serological indicators, such as liver function, blood cell analysis and serum fibrosis marker assessment, were measured in all patients. Each patient provided written informed consent at the beginning of the study. The study protocol was conducted in accordance with the provisions of the Declaration of Helsinki and its revision, and was approved by the ethical committee of Beijing YouAn Hospital, Capital Medical University (Beijing, China).

Liver biopsy. Ultrasound-guided liver biopsy examination was employed in the present study. The specimens included at least six complete portal areas and the length was $>1.5 \mathrm{~cm}$. Liver biopsy specimens were fixed in formalin and embedded in paraffin. Biopsy specimens were independently evaluated for fibrosis status, using the Metavir scoring system, by two senior pathologists who were blinded to the clinical data $(18,19)$. The fibrosis score was assessed on a five point scale (F0, no fibrosis; F1, portal fibrosis without septa; F2, few septa; F3, numerous septa without cirrhosis; and F4, cirrhosis; Fig. 1). Significant hepatic fibrosis was defined as $F \geq 2$, while severe hepatic fibrosis was defined as $\mathrm{F} \geq 3$.

HPLC-MS/MS analysis. Blood samples from patients were collected into sterile tubes using cold lithium heparin as an anticoagulant and immediately centrifuged at $4^{\circ} \mathrm{C}$ at $8,000 \mathrm{xg}$ for $10 \mathrm{~min}$. The plasma samples were stored at $-80^{\circ} \mathrm{C}$. All lipid standards were purchased from Avanti Polar Lipids (Alabaster, AL, USA). Ultra Resi-analyzed grade methanol and HPLC grade methyl-tert-butyl ether were purchased from Mallinckrodt Baker Inc. (Phillipsburg, NJ, USA). Formic acid of analytical grade was obtained from Tedia Company (Fairfield, OH, USA). Ammonium formate (purity, >99.99\%) was purchased from Sigma-Aldrich (St. Louis, MO, USA). Ultra-pure water was prepared using a Milli-Q purification system (Millipore, Bedford, MA, USA), HPLC-MS/MS was performed on an Agilent 6410B Triple Quad mass spectrometer (QQQ; Agilent Technologies Inc., Santa Clara, CA, USA) comprising a triple quadrupole MS analyzer with an electrospray ionization interface and an Agilent 1200 RRLC system. Sphingolipidomic assays were performed at the Institute of Materia Medica, Peking Union Medical College (Beijing, China) as previously described (15).

Statistical analysis. Results are expressed as the mean \pm standard deviation unless otherwise stated. Depending on data distribution, the continuous variables that differed significantly between two groups were identified by an independent samples t-test or a Mann-Whitney test. Categorical variables were compared using Pearson's $\chi^{2}$ test. The stepwise forward multivariate logistic regression analysis was performed and the P-values of entry and removal were respectively set to 0.05 and 0.1 . The diagnostic value of plasma sphingolipids with significant differences and regression model in multivariate analysis were assessed using the area under the receiver operating characteristic (ROC) curve. The negative predictive value (NPV) and positive predictive value (PPV) were also generated. Statistical analysis was performed using SPSS 
Table I. Characteristics of patients with chronic hepatitis $\mathrm{C}$ virus $(\mathrm{n}=120)$.

\begin{tabular}{|c|c|}
\hline Indicators & Value \\
\hline Age (years) & $51.33 \pm 7.33$ \\
\hline Females & $63(52.5)$ \\
\hline Males & $57(47.5)$ \\
\hline $\operatorname{ALT}(\mathrm{U} / \mathrm{l})$ & $60.42 \pm 70.88$ \\
\hline AST (U/l) & $47.94 \pm 44.30$ \\
\hline Total bilirubin $(\mu \mathrm{mol} / \mathrm{l})$ & $16.51 \pm 7.25$ \\
\hline Direct bilirubin $(\mu \mathrm{mol} / \mathrm{l})$ & $3.26 \pm 1.36$ \\
\hline Albumin (g/l) & $43.21 \pm 2.36$ \\
\hline Prealbumin (mg/l) & $186.91 \pm 176.94$ \\
\hline GGT (U/l) & $22.04 \pm 16.50$ \\
\hline Alkaline phosphatase (U/l) & $76.29 \pm 20.91$ \\
\hline White blood cell $\left(10^{9} / 1\right)$ & $5.08 \pm 1.23$ \\
\hline Red blood cell $\left(10^{12} / 1\right)$ & $4.76 \pm 0.67$ \\
\hline Hemoglobin (g/l) & $151.09 \pm 16.56$ \\
\hline Platelets $\left(10^{9} / 1\right)$ & $171.36 \pm 53.20$ \\
\hline Type III procollagen peptide $(\mu \mathrm{g} / \mathrm{l})$ & $33.84 \pm 63.73$ \\
\hline Type IV collagen $(\mu \mathrm{g} / \mathrm{l})$ & $38.62 \pm 98.14$ \\
\hline Hyaluronic acid (mg/l) & $212.72 \pm 730.42$ \\
\hline Laminin $(\mu \mathrm{g} / \mathrm{ml})$ & $37.67 \pm 29.51$ \\
\hline \multicolumn{2}{|l|}{ Fibrosis stage } \\
\hline F0 & $1(0.8)$ \\
\hline $\mathrm{F} 1$ & $55(45.8)$ \\
\hline $\mathrm{F} 2$ & $50(41.7)$ \\
\hline $\mathrm{F} 3$ & $12(10.0)$ \\
\hline $\mathrm{F} 4$ & $2(1.7)$ \\
\hline
\end{tabular}

Data are expressed as the mean \pm standard deviation, or the number of patients (percentage). ALT, alanine aminotransferase; AST, aspartate aminotransferase; GGT, $\gamma$-glutamyl transpeptidase.

version 19.0 (IBM, Armonk, NY, USA). $\mathrm{P}<0.05$ was considered to indicate a statistically significant difference.

\section{Results}

Characteristics of the untreated $\mathrm{CHC}$ cohort. Characteristics of the patients with $\mathrm{CHC}$ who were included are summarized in Table I. A total of $120 \mathrm{CHC}$ patients from the original cohort were eligible for the present study, with a mean age of 51.33 years. The average level of serum aminotransferase was mildly elevated compared with the normal range ( $<40 \mathrm{U} / \mathrm{l})$. Based on liver fibrosis staging of the liver biopsy samples (Fig. 1), F1 was assigned to 55 patients, which accounted for the largest proportion $(45.8 \%)$ of the cohort; F2 was assigned to $41.7 \%$ (50/120) of the patients; F3 was assigned to $10.0 \%(12 / 120)$ of the patients; while F0 and F4 were assigned to $1(0.8 \%)$ and $2(1.7 \%)$ patients, respectively.

Plasma sphingolipid profile in $C H C$ between $F \leq 2$ and $F>2$. Using the improved quantitative high-throughput lipidomic platform, a total of 44 plasma sphingolipids were detected in patients with $\mathrm{CHC}$ through HPLC-MS/MS. A statistically significant difference was observed between the $\mathrm{F} \leq 2$ and $\mathrm{F}>2$ groups in plasma hexosylceramide (HexCer; d18:1/12:0),
HexCer (d18:1/16:0) and HexCer (d18:1/22:0; P<0.05; Fig. 2). No statistical differences were identified in the remaining plasma sphingolipids $(\mathrm{P}>0.05)$.

Indicators associated with severe fibrosis $(F \geq 3)$ in CHC. Using univariate analysis, the routine serological indicators, alanine aminotransferase (ALT), aspartate aminotransferase (AST), albumin, prealbumin, $\gamma$-glutamyl transpeptidase (GGT), hyaluronic acid, hemoglobin, platelets, type III procollagen peptide, and type IV collagen were shown to be statistically different $(\mathrm{P}<0.05)$ between the $\mathrm{F} \leq 2$ and $\mathrm{F} \geq 3$ groups (Table II). For the multivariate analysis, HexCer (d18:1/12:0), HexCer (d18:1/16:0), HexCer (d18:1/22:0), ALT, AST, albumin, GGT, platelets, type III procollagen peptide, hyaluronic acid and type IV collagen were included in the forward stepwise logistical regression. The results demonstrated that HexCer (d18:1/12:0), ALT and AST were retained in the logistical regression equation. HexCer (d18:1/12:0), with an odds ratio (OR) value of 1.03, was associated with the presence of severe hepatic fibrosis (Table II). In addition, the area under the curve (AUC) of HexCer (d18:1/12:0), used to identify severe hepatic fibrosis, presented with 0.69 $(\mathrm{P}=0.024)$ via ROC analysis. Its NPV and PPV were $100 \%$ 
Table II. Indicators associated with severe fibrosis $(\mathrm{F} \geq 3)$ in patients with $\mathrm{CHC}$ in univariate and multivariate analysis.

\begin{tabular}{lcccc}
\hline Indicator & $\mathrm{F} \leq 2(\mathrm{n}=106)$ & $\mathrm{F} \geq 3(\mathrm{n}=14)$ & P-value $^{\mathrm{a}}$ & OR $(95 \% \mathrm{CI})$ \\
\hline Age $($ years) & $51.08 \pm 7.44$ & $53.14 \pm 6.44$ & 0.364 & \\
Females [n $(\%)]$ & $57(53.8)$ & $6(42.9)$ & & \\
Males [n $(\%)]$ & $49(46.2)$ & $8(57.1)$ & 0.442 & \\
ALT (U/l) & $51.85 \pm 49.63$ & $125.34 \pm 144.76$ & 0.001 & $0.97(0.93-1.00)$ \\
AST $(\mathrm{U} / \mathrm{l})$ & $41.27 \pm 27.97$ & $98.45 \pm 92.41$ & 0.000 & $1.08(1.02-1.14)$ \\
Total bilirubin $(\mu \mathrm{mol} / \mathrm{l})$ & $16.56 \pm 6.92$ & $16.12 \pm 9.69$ & 0.353 & \\
Direct bilirubin $(\mu \mathrm{mol} / \mathrm{l})$ & $3.23 \pm 1.26$ & $3.51 \pm 2.02$ & 0.870 & \\
Albumin $(\mathrm{g} / \mathrm{l})$ & $43.37 \pm 2.32$ & $42.00 \pm 2.42$ & 0.041 & \\
Prealbumin $(\mathrm{mg} / \mathrm{l})$ & $192.31 \pm 187.48$ & $146.01 \pm 25.94$ & 0.008 & \\
GGT $(\mathrm{U} / \mathrm{l})$ & $19.86 \pm 12.09$ & $38.51 \pm 31.28$ & 0.006 & \\
Alkaline phosphatase $(\mathrm{U} / \mathrm{l})$ & $74.66 \pm 19.08$ & $88.57 \pm 29.60$ & 0.095 & \\
White blood cell $\left(10^{9} / \mathrm{l}\right)$ & $5.15 \pm 1.22$ & $4.58 \pm 1.23$ & 0.106 & \\
Red blood cell $\left(10^{12} / \mathrm{l}\right)$ & $4.81 \pm 0.41$ & $4.34 \pm 1.60$ & 0.919 & \\
Hemoglobin $(\mathrm{g} / \mathrm{l})$ & $149.97 \pm 16.21$ & $159.57 \pm 17.35$ & 0.041 & \\
Platelets $\left(10^{9} / \mathrm{l}\right)$ & $177.25 \pm 51.16$ & $126.71 \pm 48.39$ & 0.001 & \\
Type III procollagen peptide $(\mu \mathrm{g} / \mathrm{l})$ & $28.17 \pm 34.27$ & $76.81 \pm 159.49$ & 0.036 & \\
Type IV collagen $(\mu \mathrm{g} / \mathrm{l})$ & $34.01 \pm 87.50$ & $73.51 \pm 157.65$ & 0.026 & \\
Hyaluronic acid $(\mathrm{mg} / \mathrm{l})$ & $188.73 \pm 758.21$ & $390.93 \pm 455.97$ & 0.000 & \\
Laminin $(\mu \mathrm{g} / \mathrm{ml})$ & $38.62 \pm 31.02$ & $30.52 \pm 11.73$ & 0.612 & \\
HexCer $(\mathrm{d} 18: 1 / 12: 0)(\mathrm{pmol} / \mathrm{ml})$ & $15.63 \pm 11.64$ & $26.64 \pm 27.64$ & 0.024 & $1.03(1.00-1.06)$ \\
HexCer $(\mathrm{d} 18: 1 / 16: 0)(\mathrm{pmol} / \mathrm{ml})$ & $1254.68 \pm 454.39$ & $1620.10 \pm 705.52$ & 0.016 & \\
HexCer $(\mathrm{d} 18: 1 / 22: 0)(\mathrm{pmol} / \mathrm{ml})$ & $295.10 \pm 82.53$ & $335.48 \pm 82.63$ & 0.048 & \\
\hline
\end{tabular}

Data are expressed as the mean \pm standard deviation, or the number of patients (percentage). Only plasma sphingolipids with significant differences $(\mathrm{P}<0.05)$ between groups are listed in the table. ${ }^{a} \mathrm{P}$-values were acquired using an independent-samples t-test or Mann-Whitney test depending on data distribution. CHC, chronic hepatitis C; CI, confidence interval; ALT, alanine aminotransferase; AST, aspartate aminotransferase; GGT, $\gamma$-glutamyl transpeptidase; HexCer, hexosylceramide; OR, odds ratio.
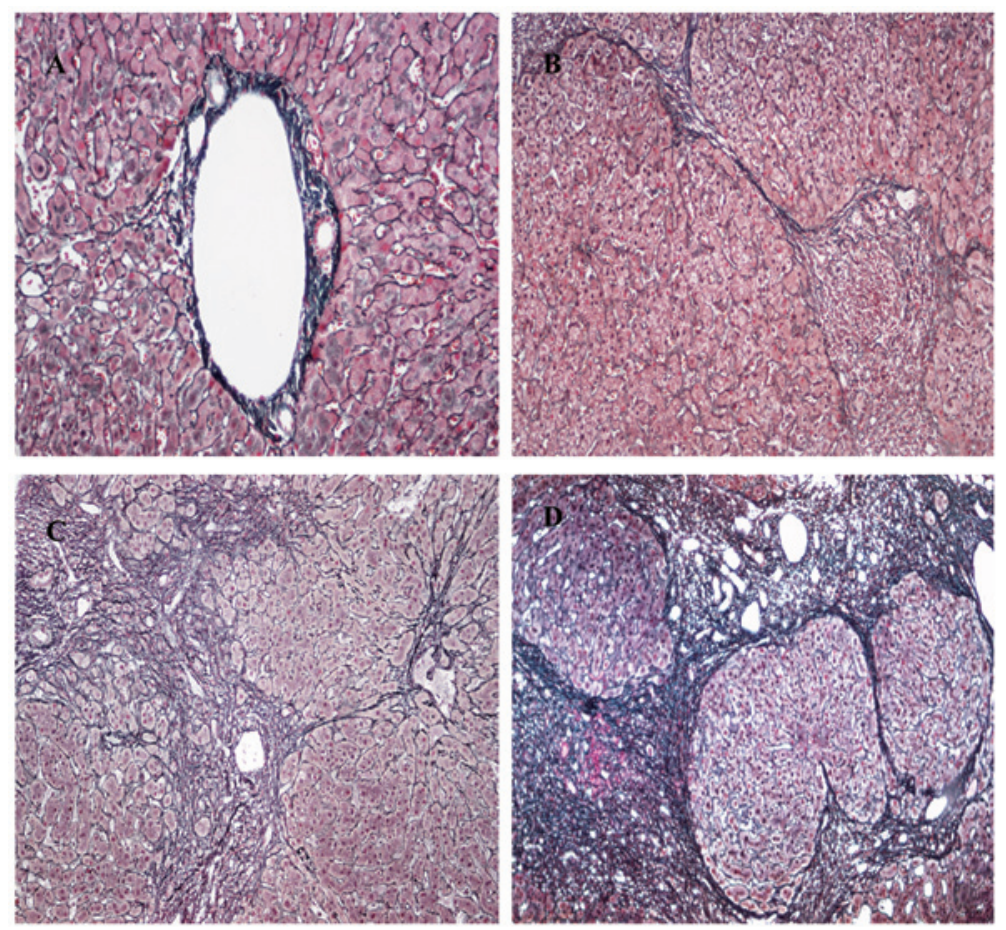

Figure 1. Liver histopathology of patients according to Metavir scoring system. (A) F1, enlargement of portal tract without septa formation (masson trichrome; magnification, x200) (B) F2, enlargement of portal tract with rare septa formation (masson trichrome; magnification, x200) (C) F3, numerous septa without cirrhosis (masson trichrome; magnification, x100) (D) F4, cirrhosis (masson trichrome; magnification, x200). 


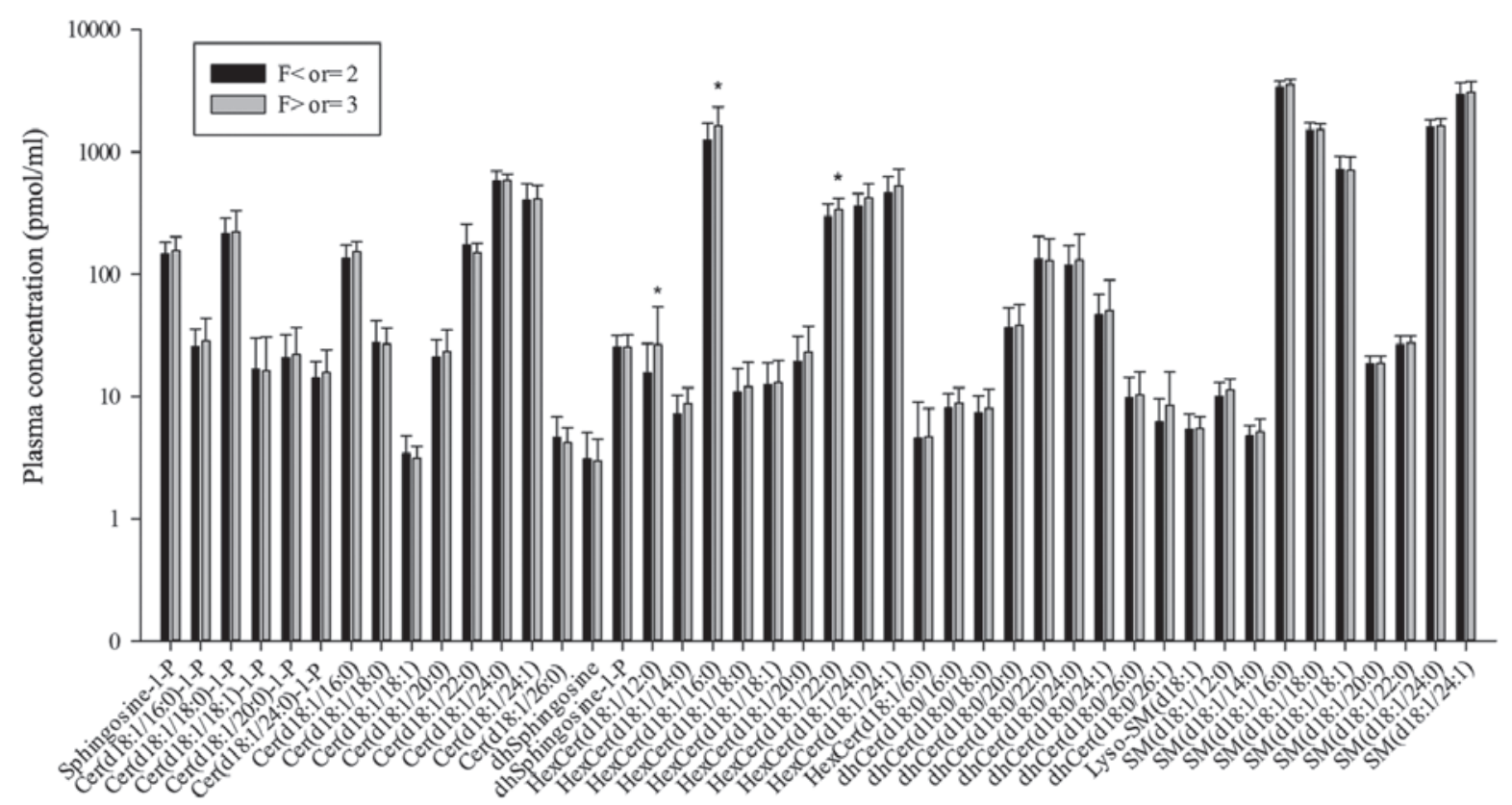

Figure 2. Plasma sphingolipids profile of patients with $\mathrm{F} \leq 2$ and $\mathrm{F} \geq 3$. Length of the column indicates the mean value and bars indicate the standard deviation. $\mathrm{P}$-values were acquired using an independent-samples t-test or Mann-Whitney test depending on data distribution. " $\mathrm{P}<0.05$, compared with $\mathrm{F} \leq 2 \mathrm{Cer}$, ceramide; dhSphingosine, dihydrosphingosine; HexCer, hexosylceramide; dhCer, dihydroceramide; SM, sphingomyelin.

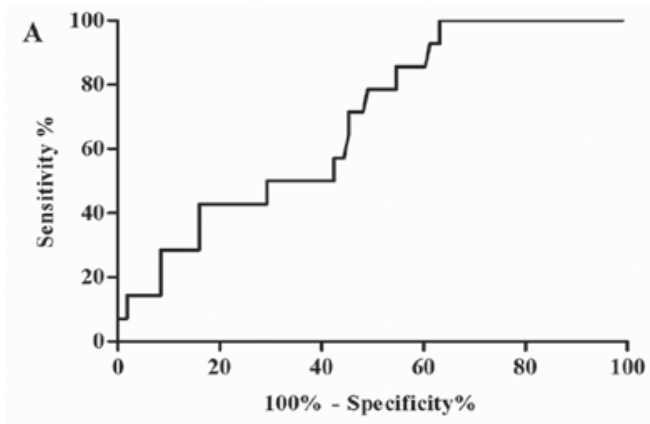

Severe fibrosis in $\mathrm{CHC}$

\begin{tabular}{cccccc}
\hline $\begin{array}{c}\text { AUC } \\
(95 \% \mathrm{CI})\end{array}$ & $\begin{array}{c}P \\
\text { value }\end{array}$ & $\begin{array}{c}\text { Sensitivity } \\
(\%)\end{array}$ & $\begin{array}{c}\text { Specificity } \\
(\%)\end{array}$ & $\begin{array}{c}\text { NPV } \\
(\%)\end{array}$ & $\begin{array}{c}\text { PPV } \\
(\%)\end{array}$ \\
$\begin{array}{c}0.69 \\
(0.56-0.81)\end{array}$ & 0.024 & 100 & 36.8 & 100 & 17.3 \\
\hline
\end{tabular}

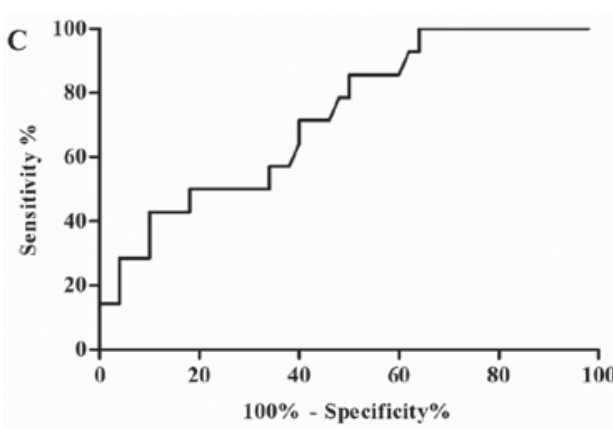

Severe fibrosis in $\mathrm{CHC}$ with significant fibrosis

\begin{tabular}{cccccc}
\multicolumn{6}{c}{ Severe fibrosis in CHC with significant fibrosis } \\
\hline $\begin{array}{c}\text { AUC } \\
(95 \% \mathrm{CI})\end{array}$ & $\begin{array}{c}P \\
\text { value }\end{array}$ & $\begin{array}{c}\text { Sensitivity } \\
(\%)\end{array}$ & $\begin{array}{c}\text { Specificity } \\
(\%)\end{array}$ & $\begin{array}{c}\text { NPV } \\
(\%)\end{array}$ & $\begin{array}{c}\text { PPV } \\
(\%)\end{array}$ \\
$\begin{array}{c}0.73 \\
(0.59-0.87)\end{array}$ & 0.009 & 100 & 36.0 & 100 & 17.2 \\
\hline
\end{tabular}

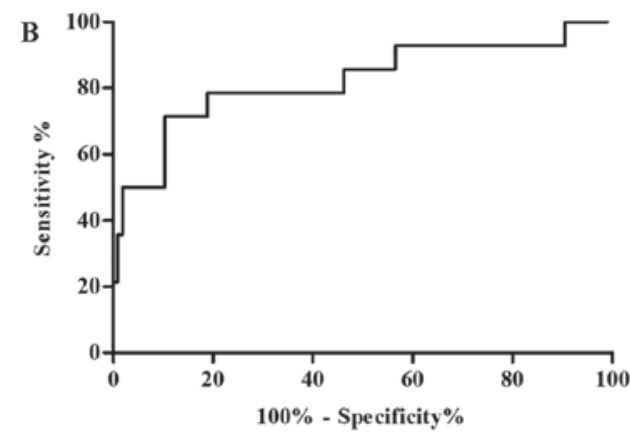

Severe fibrosis in $\mathrm{CHC}$

\begin{tabular}{cccccc}
\hline $\begin{array}{c}\text { AUC } \\
(95 \% \mathrm{CI})\end{array}$ & $\begin{array}{c}P \\
\text { value }\end{array}$ & $\begin{array}{c}\text { Sensitivity } \\
(\%)\end{array}$ & $\begin{array}{c}\text { Specificity } \\
(\%)\end{array}$ & $\begin{array}{c}\text { NPV } \\
(\%)\end{array}$ & $\begin{array}{c}\text { PPV } \\
(\%)\end{array}$ \\
0.82 & & & & & \\
$(0.68-0.96)$ & $<0.001$ & 71.4 & 89.6 & 95.9 & 47.6 \\
\hline
\end{tabular}

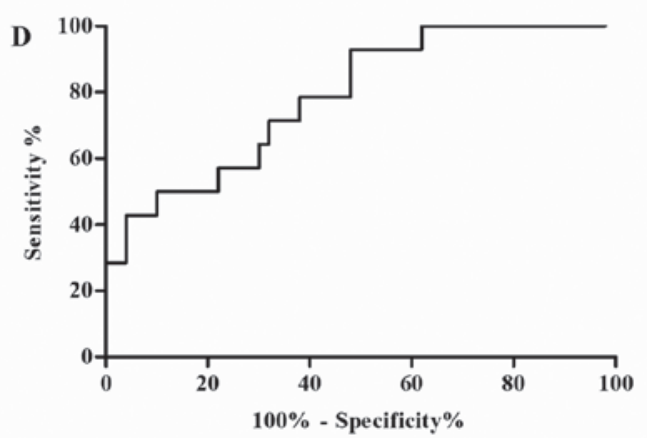

\begin{tabular}{cccccc}
\multicolumn{6}{c}{ Severe fibrosis in CHC with significant fibrosis } \\
\hline $\begin{array}{c}\text { AUC } \\
(95 \% \mathrm{CI})\end{array}$ & $\begin{array}{c}P \\
\text { value }\end{array}$ & $\begin{array}{c}\text { Sensitivity } \\
(\%)\end{array}$ & $\begin{array}{c}\text { Specificity } \\
(\%)\end{array}$ & $\begin{array}{c}\text { NPV } \\
(\%)\end{array}$ & $\begin{array}{c}\text { PPV } \\
(\%)\end{array}$ \\
$\begin{array}{c}0.79 \\
(0.66-0.92)\end{array}$ & 0.001 & 92.9 & 52.0 & 96.3 & 35.2 \\
\hline
\end{tabular}

Figure 3. ROC analysis of plasma sphingolipids. (A) performance of HexCer (d18 :1/12:0) in identifying CHC with severe fibrosis ( $\mathrm{F} \geq 3$ ). (B) Performance of logistic regression equation in identifying $\mathrm{CHC}$ with severe fibrosis $(\mathrm{F} \geq 3)$. (C) Performance of HexCer (d18:1/12:0) in identifying severe fibrosis in $\mathrm{CHC}$ with significant fibrosis ( $\mathrm{F} \geq 2$ ). (D) Performance of logistic regression equation in identifying severe hepatic fibrosis in $\mathrm{CHC}$ with significant fibrosis ( $\mathrm{F} \geq 2$ ). $\mathrm{ROC}$, receiver operating characteristic; NPV, negative predictive value; PPV, positive predictive value; CHC, chronic hepatitis C; HexCer, hexosylceramide; AUC, area under the curve. 
Table III. Indicators associated with severe fibrosis $(\mathrm{F} \geq 3)$ in patients with $\mathrm{CHC}$ with significant fibrosis $(\mathrm{F} \geq 2)$.

\begin{tabular}{|c|c|c|c|c|}
\hline Indicator & $F \geq 2(n=50)$ & $F \geq 3(n=14)$ & P-value ${ }^{a}$ & OR $(95 \% \mathrm{CI})$ \\
\hline Age (year) & $51.82 \pm 6.32$ & $53.14 \pm 6.44$ & 0.493 & \\
\hline Female $[\mathrm{n}(\%)]$ & $24(48.0)$ & $6(42.9)$ & & \\
\hline Male [n (\%)] & $26(52.0)$ & $8(57.1)$ & 0.733 & \\
\hline $\operatorname{ALT}(\mathrm{U} / \mathrm{l})$ & $64.37 \pm 65.11$ & $125.34 \pm 144.76$ & 0.012 & \\
\hline $\operatorname{AST}(\mathrm{U} / \mathrm{l})$ & $49.50 \pm 36.86$ & $98.45 \pm 92.41$ & 0.002 & $1.01(1.00-1.03)$ \\
\hline Total bilirubin $(\mu \mathrm{mol} / \mathrm{l})$ & $17.46 \pm 7.63$ & $16.12 \pm 9.69$ & 0.188 & \\
\hline Direct bilirubin $(\mu \mathrm{mol} / \mathrm{l})$ & $3.43 \pm 1.38$ & $3.51 \pm 2.02$ & 0.569 & \\
\hline Albumin (g/l) & $43.12 \pm 2.25$ & $42.00 \pm 2.42$ & 0.112 & \\
\hline Prealbumin (mg/l) & $167.00 \pm 32.80$ & $146.01 \pm 25.94$ & 0.031 & \\
\hline GGT (U/1) & $22.88 \pm 15.18$ & $38.51 \pm 31.28$ & 0.034 & \\
\hline Alkaline phosphatase (U/l) & $76.77 \pm 20.15$ & $88.57 \pm 29.61$ & 0.158 & \\
\hline White blood cell $\left(10^{9} / 1\right)$ & $4.96 \pm 1.20$ & $4.58 \pm 1.23$ & 0.306 & \\
\hline Red blood cell $\left(10^{12} / 1\right)$ & $4.85 \pm 0.38$ & $4.34 \pm 1.60$ & 0.981 & \\
\hline Hemoglobin (g/l) & $149.98 \pm 16.00$ & $159.57 \pm 17.36$ & 0.056 & \\
\hline Platelets $\left(10^{9} / 1\right)$ & $166.14 \pm 51.38$ & $126.71 \pm 48.39$ & 0.013 & \\
\hline Type III procollagen peptide $(\mu \mathrm{g} / \mathrm{l})$ & $30.26 \pm 35.97$ & $76.81 \pm 159.49$ & 0.064 & \\
\hline Type IV collagen $(\mu \mathrm{g} / \mathrm{l})$ & $42.73 \pm 120.60$ & $73.51 \pm 157.65$ & 0.129 & \\
\hline Hyaluronic acid (mg/l) & $305.56 \pm 1096.91$ & $390.93 \pm 455.97$ & 0.008 & \\
\hline Laminin $(\mu \mathrm{g} / \mathrm{ml})$ & $37.74 \pm 36.97$ & $30.52 \pm 11.73$ & 0.922 & \\
\hline HexCer (d18:1/12:0) (pmol/ml) & $13.50 \pm 6.56$ & $26.64 \pm 27.64$ & 0.009 & $1.08(0.99-1.17)$ \\
\hline HexCer (d18:1/16:0) (pmol/ml) & $1271.23 \pm 485.48$ & $1620.10 \pm 705.52$ & 0.021 & \\
\hline HexCer (d18:1/24:0) (pmol/ml) & $358.42 \pm 89.36$ & $422.80 \pm 124.25$ & 0.033 & \\
\hline
\end{tabular}

Data are expressed as the mean \pm standard deviation or the number of patients (percentage). Only plasma sphingolipids with significant difference $(\mathrm{P}<0.05)$ between the groups are listed in the table. ${ }^{a} \mathrm{P}$-values were acquired using an independent-samples t-test or Mann-Whitney test depending on data distribution. CHC, chronic hepatits C; CI, confidence interval; ALT, alanine aminotransferase; AST, aspartate aminotransferase; GGT, $\gamma$-glutamyl transpeptidase; HexCer, hexosylceramide; OR, odds ratio.

and $17.3 \%$, respectively. Additionally, the distinguishing ability of the three indicators combined logistic regression equation was also evaluated using ROC analysis. The results revealed that the AUC of the three indicators in the combined model was $0.82(\mathrm{P}<0.001)$, and the sensitivity and specificity were $71.4 \%$ and $89.6 \%$, respectively. The NPV and PPV were $95.9 \%$ and $47.6 \%$ respectively (Fig. $3 \mathrm{~A}$ and B).

Indicators associated with presence of severe fibrosis $(F \geq 3)$ in $C H C$ with significant fibrosis $(F \geq 2)$. In order to identify potential markers associated with the presence of severe hepatic fibrosis $(\mathrm{F} \geq 3)$ in patients with $\mathrm{CHC}$ who had developed significant fibrosis $(F \geq 2)$, data were analyzed using univariate and multivariate analysis (Table III). A total of 64 patients with $\mathrm{CHC}$ who had significant fibrosis were eligible for analysis. According to the results of the univariate analysis, ALT, AST, prealbumin, GGT, platelets, hyaluronic acid, HexCer (d18:1/12:0), HexCer (d18:1/16:0) and HexCer (d18:1/24:0) exhibited a significant difference $(\mathrm{P}<0.05)$ between the $\mathrm{F} \geq 2$ and $\mathrm{F} \geq 3$ groups. When all the indicators that exhibited a significant difference were included in the forward stepwise logistic regression, only HexCer (d18:1/12:0) and AST were retained in the regression equation. These variables exhibited a close association with severe fibrosis, with ORs of 1.08 and 1.01 respectively.
The ability to identify severe fibrosis in patients with $\mathrm{CHC}$ who had significant fibrosis $(F \geq 2)$ was also analyzed using the ROC curve. The results demonstrated that HexCer (d18:1/12:0) had an AUC value of $0.73(\mathrm{P}=0.009)$, with an NPV and PPV of 100 and 17.2\%, respectively. Additionally, the AUC of this equation, including HexCer (d18:1/12:0) and AST, reached $0.79(\mathrm{P}=0.001)$, and the NPV and PPV reached $96.3 \%$ and $35.2 \%$, respectively (Fig. $3 \mathrm{C}$ and D).

\section{Discussion}

Although a clinical study looking at a single sphingolipid in $\mathrm{CHC}$, in the case of S1P declining in $\mathrm{CHC}$ patients, has been previously reported, (14), to the best of our knowledge, there have been no studies that have investigated the full plasma sphingolipid profile, in order to identify biomarkers associated with the development of hepatic fibrosis induced by HCV. For the first time, to the best of our knowledge, the present study used liver biopsies and the HPLC-MS/MS method to provide a plasma sphingolipid profile of untreated patients with $\mathrm{CHC}$, with and without severe hepatic fibrosis, and demonstrated that the plasma sphingolipid, HexCer (d18:1/12:0), may have a close association with the formation of severe hepatic fibrosis in $\mathrm{CHC}$, in particular in patients with $\mathrm{CHC}$ who have developed significant fibrosis. Additionally, plasma HexCer (d18:1/12:0) 
may be used as a potential marker for patients with $\mathrm{CHC}$ with severe hepatic fibrosis.

In the present study, the plasma sphingolipid profile was analyzed in a cohort of 120 untreated patients with $\mathrm{CHC}$, who had chronic HCV infection for 20 years, which had been contracted from previous paid plasma donations. This cohort of patients with $\mathrm{CHC}$ were a suitable group to investigate as they lived in close proximity to each other and reported similar lifestyles in terms of diet and living environment. Although the incidence of severe fibrosis in this cohort was not high, it did reflect the alteration in plasma sphingolipids precisely, according to the different stages of fibrosis in the context of the treatment-naïve status. Using the improved quantitative high-throughput lipidomic methods (16), the results of the plasma sphingolipid profile showed different levels of plasma sphingolipids in patients with $\mathrm{CHC}$, with and without severe hepatic fibrosis. The altered plasma levels of HexCer (d18:1/12:0), HexCer (d18:1/16:0) and HexCer (d18:1/22:0) were observed to be associated with the presence of severe fibrosis. Following adjustment for confounding indicators, HexCer (d18:1/12:0) remained closely associated with severe fibrosis. This indicated that elevated plasma HexCer (d18:1/12:0) may be implicated in the pathogenesis of severe fibrosis in $\mathrm{CHC}$. At present, there is no direct evidence for the role of this specific glycosphingolipid in the pathogenesis of severe fibrosis due to CHC. A possible mechanism has been demonstrated in a previous study using an animal model, which reported that exogenous administration of $\alpha$-galactosylceramide accelerated carbon tetrachloride-induced liver fibrosis in vivo through the activation of invariant natural killer T cells (20). This may suggest that glycosphingolipids contribute to the progression of liver fibrosis. However, further experimental studies are required to determine the underlying mechanisms responsible for the association of HexCer (d18:1/12:0) with severe hepatic fibrosis in CHC.

The primary concern in $\mathrm{CHC}$ is the occurrence and slow evolution of fibrosis over a number of years, culminating in cirrhosis. A large scale clinical study revealed that severe fibrosis may go undetected, with few or no clinical symptoms and signs (21). Additionally, a previous study confirmed that the rate of progression to cirrhosis is accelerated in patients whose initial biopsies exhibited septal fibrosis $(F \geq 2)$ and that these individuals are at an increased risk of developing advanced cirrhosis over the ensuing decade $(22,23)$. Therefore, it is also important to examine the mechanisms underlying the evolution of fibrosis, as well as markers of severe fibrosis, which may provide insight into possible therapeutic targets to prevent the formation of severe fibrosis in patients with $\mathrm{CHC}$ who have developed septal fibrosis $(F \geq 2)$. In the present study, the plasma sphingolipids associated with severe fibrosis were evaluated in patients with $\mathrm{CHC}$ who had significant fibrosis $(\mathrm{F} \geq 2)$. It is noteworthy that HexCer (d18:1/12:0), which was closely associated with severe fibrosis in all patients with $\mathrm{CHC}$, following adjustment for confounding factors, also exhibited a correlation in a multivariate regression model, and demonstrated an association with the presence of severe fibrosis in the population of patients with $\mathrm{CHC}$ who had developed significant fibrosis. Therefore, it is hypothesized that HexCer (d18:1/12:0) may contribute to the development of advanced cirrhosis in patients with septal fibrosis $(\mathrm{F} \geq 2)$.
ROC analysis was performed in the present study. It was shown that plasma HexCer (d18:1/12:0), with an AUC of 0.69, had the diagnostic ability to distinguish severe fibrosis in $\mathrm{CHC}$. In addition, in multivariate analysis, the AUC of the indicators retained in the regression model was increased in the combined model, indicating severe fibrosis. This suggested that HexCer (d18:1/12:0) has potential as a noninvasive diagnostic indicator of severe hepatic fibrosis in CHC. This diagnostic ability may be strengthened when measurement of HexCer (d18:1/12:0) is combined with that of serum aminotransferase levels. Furthermore, the present study also identified that HexCer (d18:1/12:0) had acceptable diagnostic ability $(\mathrm{AUC}=0.73)$ with which to identify severe hepatic fibrosis in patients with $\mathrm{CHC}$ who had developed significant fibrosis. Similarly, the final regression model, including HexCer (d18:1/12:0), exhibited an improved diagnostic ability. Therefore, based on the results of the ROC analysis, HexCer (d18:1/12:0) may be utilized as a noninvasive marker to detect the presence of severe hepatic fibrosis in $\mathrm{CHC}$, in particular in patients with $\mathrm{CHC}$ who have progressed to a significant stage of fibrosis.

Issues remain concerning the precise mechanisms underlying the pathogenesis of $\mathrm{CHC}$, which were not included in the present study. In addition, $\mathrm{CHC}$ plasma donor with the same background were selected from a cohort that has had a long-term follow up. The relatively small sample size was a limitation of the present study. The diagnostic capacity of HexCer (d18:1/12:0) requires large scale clinical evaluation in the future.

In conclusion, for the first time, to the best of our knowledge, the present study analyzed the plasma sphingolipid profile in patients with $\mathrm{CHC}$, with and without severe fibrosis. Plasma HexCer (d18:1/12:0) exhibited a close association with the formation of severe fibrosis in $\mathrm{CHC}$, in particular in patients with $\mathrm{CHC}$ who had developed significant fibrosis. The present study also provided a novel insight into the molecular mechanisms underlying the development of severe fibrosis in $\mathrm{CHC}$. However, further experimental studies are required to determine the precise mechanisms underlying these associations.

\section{Acknowledgements}

The present study was supported by the National Science and Technology Key Project on 'Major Infectious Diseases such as HIV/AIDS, Viral Hepatitis Prevention and Treatment' (grant nos. 2012ZX10002004-006, 2012ZX10004904-003-001 and 2013ZX10002002-006), the Ministry of Science and Technology of the People's Republic of China (grant no. 2012ZX09301002-006), the High Technical Personnel Training Item in the Beijing Health System (grant no. 2011-3-083), the Special Scientific Research Fund for Beijing Health Development (grant no. 2011-2018-04) and YouAn Scientific Research Fund for Liver Disease and HIV/AIDS (grant no. BJYAH-2011-045). The authors would like to thank all participants involved in the present study.

\section{References}

1. Mohd Hanafiah K, Groeger J, Flaxman AD and Wiersma ST: Global epidemiology of hepatitis $C$ virus infection: new estimates of age-specific antibody to HCV seroprevalence. Hepatology 57: 1333-1342, 2013 
2. Ghany MG, Strader DB, Thomas DL and Seeff LB: Diagnosis, management, and treatment of hepatitis $C$ : an update. Hepatology 49: 1335-1374, 2009.

3. Lawson A, Hagan S, Rye K, et al: The natural history of hepatitis C with severe hepatic fibrosis. J Hepatol 47: 37-45, 2007.

4. Sebastiani G and Alberti A: How far is noninvasive assessment of liver fibrosis from replacing liver biopsy in hepatitis C? J Viral Hepat 19 Suppl 1: 18-32,2012.

5. Regev A, Berho M, Jeffers LJ, et al: Sampling error and intraobserver variation in liver biopsy in patients with chronic HCV infection. Am J Gastroenterol 97: 2614-2618, 2002.

6. Lingwood D and Simons K: Lipid rafts as a membrane-organizing principle. Science 327: 46-50, 2010.

7. Milhas D, Clarke CJ and Hannun YA: Sphingomyelin metabolism at the plasma membrane: implications for bioactive sphingolipids. FEBS Lett 584: 1887-1894, 2010.

8. Bartke N and Hannun YA: Bioactive sphingolipids: metabolism and function. J Lipid Res 50 Suppl: S91-S96, 2009.

9. Sakamoto H, Okamoto K, Aoki M, et al: Host sphingolipid biosynthesis as a target for hepatitis $\mathrm{C}$ virus therapy. Nat Chem Biol 1: 333-337, 2005 .

10. Umehara T, Sudoh M, Yasui F, et al: Serine palmitoyltransferase inhibitor suppresses HCV replication in a mouse model. Biochem Biophys Res Commun 346: 67-73, 2006.

11. Shea BS and Tager AM: Sphingolipid regulation of tissue fibrosis. Open Rheumatol J 6: 123-129, 2012.

12. Strub GM, Maceyka M, Hait NC, Milstien S and Spiegel S: Extracellular and intracellular actions of sphingosine-1-phosphate. Adv Exp Med Biol 688: 141-155, 2010.

13. Li C, Zheng S, You H, et al: Sphingosine 1-phosphate (S1P)/S1P receptors are involved in human liver fibrosis by action on hepatic myofibroblasts motility. J Hepatol 54: 1205-1213, 2011.
14. Ikeda H, Ohkawa R, Watanabe N, et al: Plasma concentration of bioactive lipid mediator sphingosine 1-phosphate is reduced in patients with chronic hepatitis C. Clin Chim Acta 411: 765-770, 2010.

15. Qu F, Wu CS, Hou JF, Jin Y and Zhang JL: Sphingolipids as new biomarkers for assessment of delayed-type hypersensitivity and response to triptolide. PLoS One 7: e52454, 2012.

16. Qu F, Zheng SJ, Wu CS, Jia ZX, Zhang JL and Duan ZP: Lipidomic profiling of plasma in patients with chronic hepatitis C infection. Anal Bioanal Chem 406: 555-564, 2014.

17. Hepatotogy Branch, Infectious and Parasitology branch, Chinese Medical Association: Guideline of prevention and treatment of hepatitis C. Zhonghua Yu Fang Yi Xue Za Zhi 38: 210-215, 2004

18. Group TFMCS Intraobserver and interobserver variations in liver biopsy interpretation in patients with chronic hepatitis $\mathrm{C}$. Hepatology 20: 15-20, 1994.

19. Bedossa P and Poynard T: An algorithm for the grading of activity in chronic hepatitis C. The METAVIR Cooperative Study Group. Hepatology 24: 289-293, 1996.

20. Park O, Jeong WI, Wang L, et al: Diverse roles of invariant natural killer $\mathrm{T}$ cells in liver injury and fibrosis induced by carbon tetrachloride. Hepatology 49: 1683-1694, 2009.

21. Di Bisceglie AM: Natural history of hepatitis C: its impact on clinical management. Hepatology 31: 1014-1018, 2000.

22. Yano M, Kumada H, Kage M, et al: The long-term pathological evolution of chronic hepatitis C. Hepatology 23: 1334-1340, 1996.

23. Marcellin P, Asselah T and Boyer N: Fibrosis and disease progression in hepatitis C. Hepatology 36: S47-S56, 2002. 